\title{
Substituição da gordura hidrogenada por óleo de soja na elaboração de pães de linhaça e avaliação da aceitabilidade
}

\author{
Substitution of hydrogenated fat by soy oil in the elaboration of \\ flaxseed bread and evaluation of sensory acceptance
}

\author{
Valéria Alcântara Santos CALDERELLI ${ }^{1}$, Marta de Toledo BENASSI ${ }^{2}$, Graciette MATIOLI ${ }^{3 *}$
}

\begin{abstract}
Resumo
A semente de linhaça apresenta cerca de $60 \%$ de ácidos graxos ômega-3, os quais auxiliam na prevenção de células malignas, enquanto que os ácidos graxos trans podem levar a implicações nutricionais negativas. Foi objetivo do trabalho elaborar uma nova formulação de pão de linhaça com a substituição da gordura hidrogenada por óleo de soja. Foi verificada a aceitação e realizada a quantificação dos ácidos graxos trans e ácidos graxos ômega-3 e ômega-6 nos produtos padrão e modificado. Foram, ainda, avaliados parâmetros instrumentais de cor e textura. As análises físico-químicas e de composição centesimal seguiram as metodologias do Instituto Adolf Lutz e AOAC. Para determinação de ácidos graxos utilizou-se cromatografia gasosa. A nova formulação apresentou maiores teores de ácidos graxos ômega-6 e ômega-3 e de poliinsaturados em relação ao padrão. Inversamente, a quantidade de ácidos graxos trans foi significativamente maior no pão padrão. Não foi observada diferença na cor de casca, mas a formulação com óleo de soja apresentou miolo mais amarelado e maior maciez que o padrão. O pão formulado com óleo de soja apresentou boa aceitação sensorial.
\end{abstract}

Palavras-chave: ácidos graxos trans; ácidos graxos ômega-3; Linum usitatissimum; aceitabilidade; cor; textura.

\begin{abstract}
Flaxseed presents about $60 \%$ omega- 3 fatty acids, which aid in the prevention of the growth of cancerous cells, while trans fatty acids can lead to negative nutritional implications. This work aimed at elaborating a novel formulation of flaxseed bread with the substitution of hydrogenated fat by soy oil. Sensory acceptance was verified and the quantification of trans fatty acids and omega-3 and omega- 6 fatty acids was accomplished in the modified and standard products. Instrumental parameters of color and texture were evaluated. Physical-chemical analysis and proximate composition were carried out according to Adolfo Lutz Institute and AOAC methodology. Gas chromatography was used to determine fatty acid composition. The novel formulation presented higher quantities of polyunsaturated fatty acids, omega- 6 and omega-3 fatty acids, in relation to the standard sample. On the other hand, the amount of trans fatty acids was significantly higher in the standard bread. No difference was observed in crust color; however, the formulation with soy oil presented a more yellowish crumb and greater softness than the standard. The bread formulated with soy oil presented good sensory acceptance.
\end{abstract}

Keywords: trans fatty acids; omega-3 fatty acids; Linum usitatissimum; sensory acceptance; color; texture.

\section{Introdução}

Nas últimas décadas, estudos sobre a função dos óleos e gorduras na nutrição humana têm enfatizado a importância da ingestão de ácidos graxos ômega-3, a redução de saturados e, mais recentemente, o controle da ingestão de ácidos graxos trans (ASCHERIO, 1999). A Food and Drug Administration (FDA) sugeriu, em 1999, que o teor de ácidos graxos trans fosse incluído nos rótulos dos produtos alimentícios (FDA, 2006). Em dezembro de 2003, foi publicada no Brasil uma resolução que obriga a rotulagem nutricional, dentre os nutrientes obrigatórios estão as gorduras trans (BRASIL, 2003).

Os isômeros trans são formados no processo de fritura, assim como no refino e hidrogenação de óleos (JUDD, 1994; SEBEDIO, 1996). A hidrogenação parcial dos óleos vegetais poliinsaturados leva a um aumento dos ácidos graxos trans, gerando gorduras com ponto de fusão mais alto, maior plasticidade e estabilidade oxidativa que os óleos originais (EMKEN, 1984; HUI, 1996). As gorduras parcialmente hidrogenadas são as maiores fontes destes ácidos, sendo utilizadas para dar estabilidade no sabor e textura e aumentar a vida de prateleira dos alimentos (FDA, 2003; FELDMAN, 1996). São encontradas em muitos alimentos processados, como margarinas, massas e recheios de biscoitos, formulações para sopas e cremes, produtos de panificação e frituras (MARTIN, 2005; SANIBAL; MANCINI FILHO, 2004). Estão, ainda, presentes naturalmente, em pequenas quantidades, na gordura do leite $\mathrm{e}$ outras (LARQUE; ZAMORA; GIL, 2001). Com a nova legislação em vigor (BRASIL, 2003) tem-se pensado na substituição da gordura hidrogenada, o que pode acarretar problemas na

Recebido para publicação em 30/5/2007

Aceito para publicação em 13/1/2008 (002509)

1 Pós-graduação em Ciências da Saúde, Universidade Estadual de Maringá - UEM

2 Departamento de Tecnologia de Alimentos e Medicamentos, Universidade Estadual de Londrina - UEL

${ }_{3}^{3}$ Departamento de Farmácia e Farmacologia, Universidade Estadual de Maringá - UEM, Av. Cerro Azul, 1467, Zona 2, CEP 87010-000, Maringá - PR, Brasil,

E-mail: gmatioli@uem.br

${ }^{*}$ A quem a correspondência deve ser enviada 
textura dos alimentos. Kenny et al. (2000) verificaram que alguns compostos ajudam na maciez e outros apresentam ação deletéria dependendo do ingrediente empregado e do tempo de armazenamento.

A gordura trans atua no organismo como a gordura saturada, elevando as lipoproteínas de baixa densidade (LDL), aumentando o colesterol e os riscos de doenças cardiovasculares (ASCHERIO et al., 1999; COVINGTON, 2004; JUDD et al., 1994; LOUHERANTA et al., 1999). Tem sido correlacionada com a etiologia de várias disfunções metabólicas, como a inibição do metabolismo de ácidos graxos essenciais (LARQUE; ZAMORA; GIL, 2001; MARTIN et al., 2005). Maior atenção tem sido dada ao consumo de ácidos graxos saturados, entretanto, estudos demonstram que a ingestão de altos níveis de gordura trans promove um aumento mais significativo na razão LDL/HDL-colesterol e, conseqüentemente, um efeito mais adverso à saúde (ASCHERIO et al., 1999; VALENZUELA; MORGADO, 1999).

A Organização Mundial da Saúde (OMS) preconiza o controle no consumo de ácidos graxos trans, com vistas à prevenção e tratamento de doenças coronarianas (WHO, 2006). $\mathrm{O}$ aumento do consumo de alimentos com níveis elevados de ácidos graxos trans pode, além das implicações nutricionais relatadas, ter como conseqüência direta a redução da ingestão de ácidos graxos essenciais, favorecendo o desenvolvimento de síndromes relacionadas à deficiência destes ácidos graxos (MARTIN et al., 2006).

Os ácidos graxos essenciais estão divididos em dois grupos: os da família ômega-3 (ácido linolênico) e ômega-6 (ácido linoléico). Estes compostos são encontrados em peixes de água fria, óleos vegetais, semente de linhaça, nozes e alguns vegetais. Os ácidos graxos ômega-3 apresentam dois derivados muito importantes: o ácido eicosapentaenóico (EPA) e o ácido docosahexaenóico (DHA). O EPA está associado à prevenção de doenças cardiovasculares, pela redução dos níveis de triglicérides e colesterol sanguíneo (JUMP, 2002). O DHA apresenta capacidade de prevenir doenças cardíacas, reduzir a taxa de triglicerídeos, além de ser importante no desenvolvimento da função visual e cerebral (CONNOR, 2000; GIBSON; MAKRIDES, 2000; KRIS-ETHERTON; HARRIS; APPEL, 2002; MACHADO; SANTIAGO, 2001; VAINIO; MUTANEM, 2000; VALENZUELA; NIETO, 2003).

A linhaça (Linum usitatissimum; Linaceae) é uma semente marrom escura, que na alimentação é usada como complemento no preparo de pães e bolos (HALL et al., 2005). O ácido ômega-3 presente em cerca de $60 \%$, faz com que a semente seja a maior fonte vegetal deste composto. O ácido graxo ômega-6 também está presente (CUNNANE et al., 1993; MAYES, 1996). Portanto, a ingestão de linhaça proporciona níveis adequados de ácidos graxos poliinsaturados, atuando na prevenção e modulação de doenças cardíacas e auto-imunes, de câncer de mama, próstata e cólon e artrite reumatóide (CUNNANE et al., 1993; JUMP, 2002; KRIS-ETHERTON; HARRIS; APPEL, 2002; THURNHAM, 1999). A elaboração de alimentos com linhaça vem contribuir para o consumo desta semente como fonte de alimento funcional (CUNNANE et al., 1995; HALL et al., 2005), contudo, o uso de gordura hidrogenada na fabricação destes pães não é saudável e a implantação de processamentos tecnológicos adequados visando reduzir a formação de ácidos graxos trans será, provavelmente, uma conseqüência direta da exigência legal da sua substituição (AUED-PIMENTEL et al., 2003).

Considerando a atual tendência na utilização de ingredientes com propriedades funcionais e a necessidade de reduzir o consumo de ácidos graxos trans na dieta, este trabalho teve por objetivo verificar a viabilidade da substituição da gordura hidrogenada por óleo de soja numa formulação de pão de linhaça, avaliando as características sensoriais e nutricionais dos dois produtos.

\section{Material e métodos}

\subsection{Produção dos pães de linhaça}

Os pães foram manufaturados em batedeira industrial (Perfecta, Mod. RS-20, Curitiba/PR - Brasil) como segue: a aglutinação dos ingredientes foi feita com a mistura da gordura hidrogenada (Coamo, Campo Mourão/PR - Brasil) ou óleo de soja (Cocamar, Maringá/PR - Brasil) com os ingredientes secos das formulações (Tabela 1). Adicionou-se linhaça em grão (Jasmine, Curitiba/PR - Brasil) e, posteriormente, o fermento, seguido da água. Após a enformagem, a cocção foi realizada em forno (Perfecta, Mod. Modulare, Curitiba/PR - Brasil) numa temperatura de $170{ }^{\circ} \mathrm{C} / 30$ minutos. Após o resfriamento, os pães foram fatiados em fatiadeira industrial, com uma espessura de 1,5 cm (Perfecta, Mod. RDS-09, Curitiba/PR - Brasil) e acondicionados em embalagens plásticas. Neste trabalho, foi considerado referência o pão de linhaça comumente comercializado e elaborado com gordura hidrogenada. Na nova formulação a gordura hidrogenada foi substituída por óleo de soja. A substituição da gordura por óleo vegetal foi proporcional à receita padrão, que corresponde a $4 \%$ da formulação.

\subsection{Composição centesimal aproximada}

As análises de teor de umidade, cinzas e proteína foram realizadas conforme técnicas da AOAC (1998). O fator 6,25 foi considerado na determinação da proteína. Os lipídios totais foram determinados segundo Bligh e Dyer (1959), na proporção

Tabela 1. Formulação final do pão de linhaça padrão e da nova formulação com óleo e soja, em gramas por $100 \mathrm{~g}$ do produto.

\begin{tabular}{lcc}
\hline \multicolumn{1}{c}{ Ingredientes } & $\begin{array}{c}\text { Pão de linhaça } \\
\text { padrão }\end{array}$ & $\begin{array}{c}\text { Nova formulação } \\
\text { com óleo de soja }\end{array}$ \\
\hline Farinha de trigo $(\mathrm{g})$ & 47 & 47 \\
Água $(\mathrm{g})$ & 27 & 27 \\
Linhaça (g) & 6 & 6 \\
Fibra de trigo $(\mathrm{g})$ & 4 & 4 \\
Fermento biológico $(\mathrm{g})$ & 4 & 4 \\
Gordura vegetal $(\mathrm{g})$ & 4 & - \\
Óleo de soja $(\mathrm{g})$ & - & 4 \\
Açúcar $(\mathrm{g})$ & 3 & 3 \\
Sal $(\mathrm{g})$ & 0,7 & 0,7 \\
Reforçador $(\mathrm{g})$ & 0,5 & 0,5 \\
Antimofo $(\mathrm{g})$ & 0,2 & 0,2 \\
\hline
\end{tabular}


de metanol:clorofórmio:água, 2:2:1,8 (v/v/v), respectivamente. As análises de fibras e composição mineral fixa (sódio) foram realizadas pelos métodos do Instituto Adolf Lutz (2005). Os teores de carboidratos foram estimados por diferença.

\subsection{Análise cromatográfica dos ésteres metílicos de ácidos graxos}

A transesterificação dos ácidos graxos dos lipídios totais foi realizada segundo procedimento de Joseph e Ackman (1992). Os ésteres de ácidos graxos foram quantificados em cromatógrafo a gas (Varian, modelo AA 3380), equipado com um detector de ionização de chama e coluna capilar de sílica fundida $\mathrm{CP}$ (Select FAME-CP-7420) (100 m x, 0,25 mm de diâmetro interno e $0,25 \mu \mathrm{m}$ de cianopropil). A vazão de $\mathrm{H}_{2}$ (gás de arraste) foi de $1,0 \mathrm{~mL} /$ minuto, com $30 \mathrm{~mL} /$ minuto de $\mathrm{N}_{2}$ e 30 e $300 \mathrm{~mL} /$ minuto, respectivamente, para a chama do detector. $\mathrm{O}$ volume injetado foi de $1,0 \mu \mathrm{L}$, utilizando split 1:80. As temperaturas do injetor e detector foram de 220 e $240{ }^{\circ} \mathrm{C}$, respectivamente, enquanto a temperatura da coluna foi de $165^{\circ} \mathrm{C}$, durante 18 minutos, e elevada a $235^{\circ} \mathrm{C}$ com taxa de $4^{\circ} \mathrm{C} /$ minuto, mantida por 25 minutos. A identificação dos ácidos graxos foi efetuada através da comparação com padrões (Sigma-EUA), co-cromatografia e valores de ECL (Equivalente Chain Lenght), a partir dos tempos de retenção corrigidos das amostras. As concentrações foram determinadas através da integração das áreas dos picos pelo software Varian Workstation Star, versão 5.0. Os resultados foram expressos em miligramas de ácidos graxos por grama de lipídios totais, através da padronização interna e utilizando padrões de ésteres metílicos de ácidos graxos da marca Sigma com 99\% de pureza. O metil éster dos ácidos tricosoanóico (23:0 metil tricosonoato) foi utilizado como padrão interno por não estar presente nas amostras. Os cálculos foram realizados segundo método de Joseph e Ackman (1992).

\subsection{Análise instrumental de cor}

A determinação de cor foi feita na casca e no interior das amostras de pão, utilizando-se um colorímetro portátil Minolta CR10 (local). As medidas foram realizas com a seguinte especificação do equipamento: área de leitura $8 \mathrm{~mm}$ iluminante CIE D 65 (luz natural do dia), colocado num ângulo de $8^{\circ} \mathrm{e}$ observador padrão $\mathrm{CIE} 10^{\circ}$. Foram determinados os valores de luminosidade, componente vermelho-verde e componente amarelo-azul (L*, $\left.\mathrm{a}^{\star}, \mathrm{b}^{*}\right)$. (ABNT, 1992; LAWLESS; HEYMANN, 1998). Foram realizadas 10 repetições de cada medida.

\subsection{Análise instrumental de textura}

Para as medidas de textura utilizou-se um texturômetro TA-XT2 (Stable Micro Systems, Surrey, Inglaterra), conforme metodologia da AACC (método padrão 74-09) (ABI, 2007). As condições de operação do equipamento empregadas foram: sensor "probe" cilíndrico de acrílico com diâmetro de uma polegada, velocidade de teste $2,0 \mathrm{~mm} / \mathrm{s}$, distância $10,0 \mathrm{~mm}$ e força $0,10 \mathrm{~N}$. As três primeiras fatias das extremidades de cada pão foram excluídas do teste. Para análise de compressão foram utilizadas duas fatias empilhadas para cada medida e foram realizadas dez medidas para cada pão. Foi determinado o valor de dureza $(\mathrm{N})$.

\subsection{Análise estatística}

Foi empregado um delineamento inteiramente casualizado. Os resultados foram submetidos à análise de variância (ANOVA), considerando-se a amostra padrão pão de linhaça e a nova formulação com óleo como única causa de variação e o teste de Tukey a 5\% de probabilidade, através do software Statistica 6.0/2001 (Stat Soft, Inc. Tulsa, OK, EUA) (STATISTICA, 2001).

\subsection{Análise sensorial}

Os testes foram realizados em laboratório, à temperatura ambiente, em cabines individuais com luz branca, nos horários entre 9:00 e 12:00 horas e entre 14:00 e 17:00 horas. A ordem de apresentação das amostras foi aleatorizada para cada sessão.

Para verificar a existência de diferença sensorial entre as amostras pelo uso de gordura hidrogenada ou óleo de soja, foi utilizado um teste triangular empregando-se 36 provadores. As fatias de pães (20 g) foram cortadas ao meio em um corte longitudinal e colocadas em pratos codificados com número de três dígitos. Entre as provas foi oferecida água. Com o mesmo grupo de provadores, foi também realizado teste triangular de aparência com os pães em suas embalagens originais, como seriam comercializados, codificadas com 3 dígitos. Para a análise dos resultados, foi utilizado o teste de Qui-Quadrado (STONE; SIDEL, 1993), considerando-se o nível de significância de 5\%.

Para verificar a aceitação da nova formulação, 54 consumidores avaliaram amostras de pão elaborado com óleo de soja. Utilizou-se uma escala hedônica estruturada de 7 pontos, com termos verbais nas extremidades e meio (OLIVEIRA et al., 2004). Também foi solicitado aos provadores que citassem as características que mais gostaram e o que menos gostaram na amostra analisada.

\section{Resultados e discussão}

O pão de linhaça padrão e a nova formulação com óleo apresentaram a seguinte composição centesimal média (\%): umidade e voláteis $31,16 \pm 0,05$, resíduo mineral 1,65 $\pm 0,04$, proteína bruta $8,26 \pm 0,02$, lipídios $6,73 \pm 0,03$, fibra $3,77 \pm 0,04$, sódio (mg.100 g-1) 320,43 \pm 0,18 e carboidrato total (calculado por diferença) 48,41\%. O valor calórico médio foi de $287 \pm 0,14 \mathrm{kcal} .100 \mathrm{~g}^{-1}$.

A composição de ácidos graxos da linhaça in natura, do óleo de soja, do pão de linhaça padrão e da nova formulação com óleo de soja está apresentada na Tabela 2. Em relação aos ácidos graxos saturados e monoinsaturados, não houve diferença significativa entre as formulações estudadas. Quanto aos ácidos graxos poliinsaturados, a nova formulação com óleo de soja apresentou teores significativamente maiores que o pão de linhaça padrão. O inverso ocorreu para os ácidos graxos trans, devido à presença da gordura hidrogenada na formulação padrão. A nova formulação com óleo de soja também apresentou concentração significativamente maior de ácido graxo ômega-6 
Tabela 2. Composição de ácidos graxos da linhaça in natura, do óleo de soja, dos pães de linhaça padrão e da nova formulação com óleo de soja, em porcentagem (gramas por $100 \mathrm{~g}$ do produto) ${ }^{1}$.

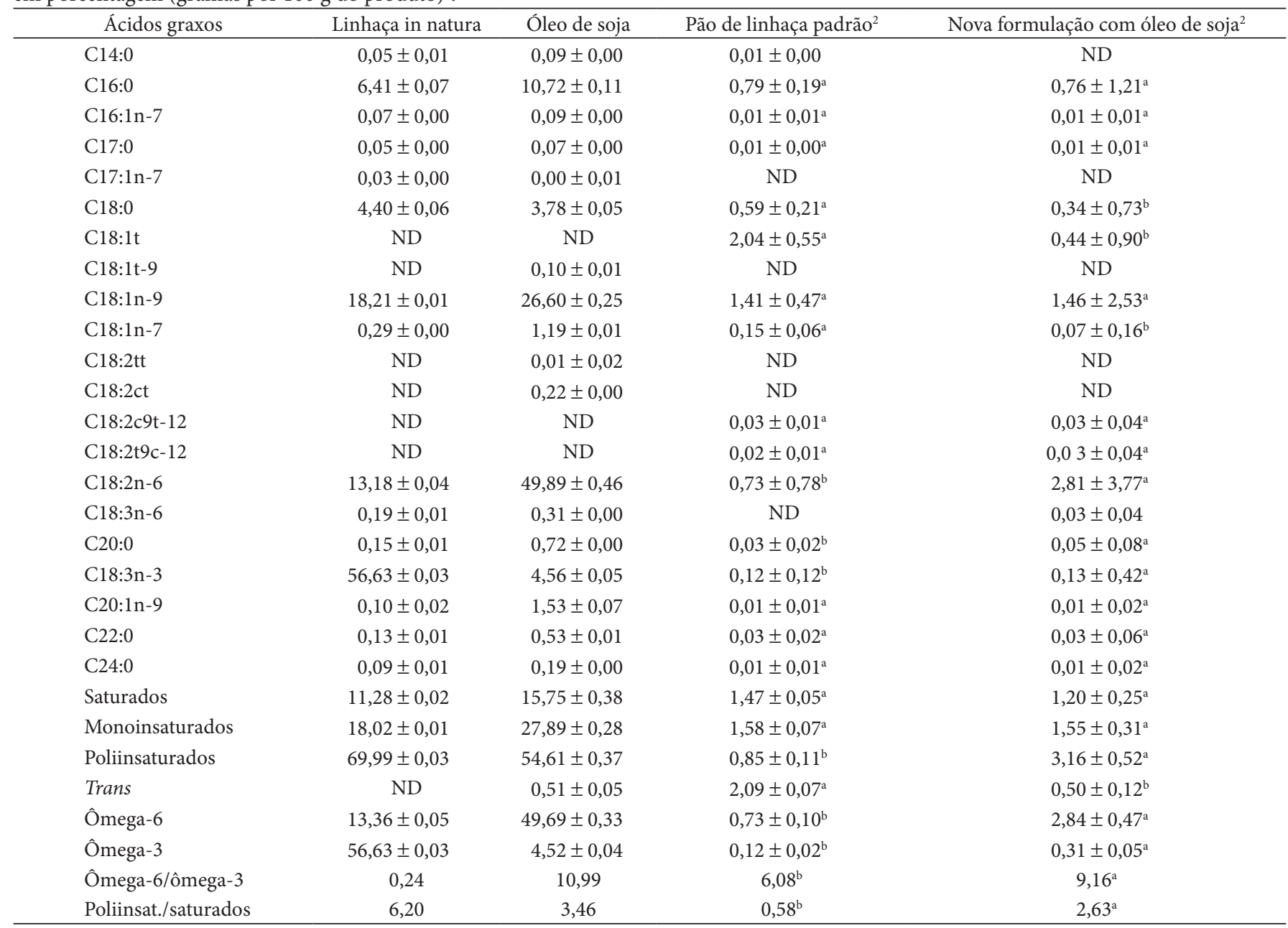

${ }^{1}$ Médias de três repetições analíticas \pm desvio padrão; ${ }^{2}$ Letras diferentes na mesma linha indicam diferenças estatísticas significativas entre as amostras ( $\left.\mathrm{p} \leq 0,05\right)$; e ND = não detectado.

e ácido graxo ômega-3, bem como valores mais altos de razão ômega-6/ômega-3 que o pão de linhaça padrão. Portanto, nota-se que o uso de gordura hidrogenada nos produtos de panificação contribui para maior consumo de ácidos graxos trans, sendo a nova formulação com óleo de soja, proposta neste trabalho, com características mais saudáveis, especialmente no que se refere aos ácidos graxos poliinsaturados e ácidos graxos ômega.

Segundo as recomendações do Department of Health (1994), a razão entre as somatórias dos ácidos graxos ômega-6 e ômega-3 deve ser no máximo 4 e a razão entre as somatórias dos ácidos graxos poliinsaturados e saturados deve ser maior que 0,45 , para serem consideradas saudáveis em relação às doenças cardíacas. Portanto, verifica-se na Tabela 2 que tanto o pão de linhaça padrão quanto a nova formulação com óleo de soja se mantiveram dentro do parâmetro mencionado para ácidos graxos poliinsaturados e saturados.

Foi determinado o valor nutricional por porção de cada amostra e o valor diário recomendado (VD\%), baseado em 2000 calorias/dia, de acordo com Resolução no 360/2003, da ANVISA (2003), conforme Tabela 3. Considerando que esta
Resolução determina que um produto seja declarado isento de ácidos graxos trans quando a quantidade destes for igual ou menor que $0,2 \mathrm{~g}$ por porção, observou-se que somente a nova formulação com óleo de soja se classifica em produto sem gordura trans. O fato da nova formulação apresentar $0,2 \mathrm{~g}$ por porção de ácidos graxos trans (Tabela 3) é devido ao óleo de soja apresentar estes ácidos graxos oriundos do seu refino (MARTIN et al., 2006). Segundo Sirinivasan et al. (2006), a OMS define que o consumo de ácidos graxos trans deve ser menor que $1 \%$ das energias diárias. Portanto, observando a Tabela 3, conclui-se que para o consumidor seguir a recomendação acima poderá consumir até 4 fatias do pão de linhaça padrão e o dobro de fatias da nova formulação. Isto vem confirmar a característica nutricional positiva desta formulação.

A Food and Agriculture Organization, FAO (2006) recomenda, para ingestão diária, uma razão de ácidos graxos ômega-6:ômega-3 entre 5:1 e 10:1. Para alguns órgãos é mais eficaz estabelecer níveis de ingestão adequada para os ácidos graxos individualmente do que determinar a razão entre eles. De acordo com a International Society for the Study of Fatty Acids and Lipids (ISSFAL), a ingestão adequada para o adulto de 
ácido graxo ômega-6 é de 4,44 g/dia (ou 2\% do total de energia ingerida), e de ácido graxo ômega-3 é de 2,22 g/dia (ou 1\% do total de energia ingerida) (ISSFAL, 2006). Segundo a Food and Nutrition Board Institute of Medicine, as recomendações de ingestão diária para adultos são de 5 a 10\% e 0,6 a 1,2\% do total de energia para ácidos graxos ômega-6 êmega-3, respectivamente (DRI's, 2002). Já a OMS, recomenda uma ingestão de 5 a $8 \%$ para ácido graxo ômega-6 e 1 a $2 \%$ de ácido graxo ômega-3, em relação ao consumo energético diário (SRINIVASAN; IRZ; SHANKAR, 2006). Portanto, ao considerar a média percentual das recomendações acima para a ingestão diária, ou seja, 5\% para ácido graxo ômega-6 e 1\% para ácido graxo ômega-3, observou-se que a nova formulação com óleo de soja apresentou em sua porção (2 fatias de pão) os melhores valores diários de recomendação, sendo 14\% para ácido graxo ômega-6 e 9\% para ácido graxo ômega-3 (Tabela 3). Para atingir 100\% da ingestão diária recomendada para ácido graxo ômega-6, deve-se consumir 14 fatias da nova formulação com óleo de soja, sendo necessário o consumo de 50 fatias para o pão de linhaça padrão. Em relação ao ácido graxo ômega-3, deve-se consumir 22 fatias da nova formulação com óleo de soja e 67 fatias para o pão de linhaça padrão.

Conforme a Portaria n ${ }^{\circ} 27 / 1998$, ANVISA (1998), produtos com teores de fibras de no mínimo $3 \mathrm{~g} .100 \mathrm{~g}^{-1}$ podem receber o atributo "fonte de fibra". Observando a Tabela 3, os teores de fibras da amostra padrão e da nova formulação com óleo de soja encontram-se em quantidades que permitem o uso no rótulo do atributo acima citado.

Os resultados da análise instrumental de cor estão apresentados na Tabela 4. A substituição de gordura hidrogenada por óleo de soja não afetou os parâmetros de cor da casca, contudo, a nova formulação com óleo de soja apresentou o miolo com cor mais amarelada em relação ao pão de linhaça padrão. Quanto à textura, as amostras diferiram significativamente entre si, sendo que o pão de linhaça padrão apresentou textura mais dura em relação à nova formulação com óleo de soja. Portanto, a mudança na composição química da nova formulação, ou seja, a substituição da gordura hidrogenada por óleo de soja proporcionou um pão de maciez significativamente maior em relação ao padrão. A afirmação de Vosey et al. (1976), de que os fatores responsáveis pelas variações naturais de textura no alimento são as diferenças na composição química ou forças físicas que atuam sobre ele, vem confirmar os resultados de textura obtidos.

Nos testes triangulares, os provadores observaram diferença significativa (5\%) na aparência das amostras quando embaladas, mas não detectaram diferença sensorial significativa no global, ou seja, não perceberam diferença no uso de gordura hidrogenada ou de óleo de soja no momento do consumo.

A nova formulação foi bem aceita, recebendo nota média de 5,6 numa escala de 7 pontos. Quando foi solicitado aos provadores que citassem os atributos que mais gostaram para a nova formulação com óleo de soja, destacou-se a aprovação da maciez e textura do produto (60\%), sendo ainda citado o sabor (43\%). Em relação aos atributos que menos gostaram, poucas foram as citações e estas não tiveram representatividade junto aos provadores.

Pinto et al. (2003) avaliaram parâmetros da qualidade da batata frita em gordura hidrogenada, óleo de girassol e óleo de milho. Os resultados obtidos na extração de gordura da batata frita foram confirmados pela avaliação sensorial, ou seja, a batata frita em gordura hidrogenada obteve menor crocância e maior residual de gordura. Devido à dificuldade de encontrar trabalhos com o mesmo curso de investigação aqui realizado, ou seja, com substituição de gordura hidrogenada por óleo vegetal em panificação, os dados desta pesquisa não foram passíveis de

Tabela 3. Valor nutricional por porção de cada amostra e valor diário recomendado (VD\%), baseado em 2000 calorias/dia ${ }^{1}$.

\begin{tabular}{|c|c|c|c|c|}
\hline Determinações & $\begin{array}{l}\text { Pão de linhaça padrão } \\
\text { (porção } 52 \text { g } 2 \text { fatias) }\end{array}$ & $\mathrm{VD}(\%)$ & $\begin{array}{l}\text { Nova formulação com óleo de } \\
\text { soja (porção } 53 \text { g } 2 \text { fatias) }\end{array}$ & $\mathrm{VD}(\%)$ \\
\hline Calorias (kcal) & 150 & 8 & 150 & 8 \\
\hline Carboidratos (g) & 25 & 8 & 25 & 8 \\
\hline Ácidos graxos totais (g) & 3,5 & 6 & 3,5 & 6 \\
\hline Ácidos graxos ômega-6 (g) & 0,4 & 4 & 1,5 & 14 \\
\hline Ácidos graxos trans $(\mathrm{g})$ & $1,1 \mathrm{~g}$ & * & 0,2 & * \\
\hline Fibras $(g)$ & 2 & 8 & 2 & 8 \\
\hline Sódio (mg) & 156 & 7 & 181 & 8 \\
\hline
\end{tabular}

${ }^{1}$ De acordo com resolução 360/2003 do Ministério da Saúde ${ }^{6} ; e^{*}$ Valor diário não estabelecido.

Tabela 4. Parâmetros de textura, cor da casca e do miolo do pão de linhaça padrão e da nova formulação com óleo de soja.

\begin{tabular}{|c|c|c|c|c|c|c|c|}
\hline \multirow[t]{2}{*}{ Amostras de pão } & \multicolumn{3}{|c|}{ Casca } & \multicolumn{3}{|c|}{ Miolo } & \multirow[t]{2}{*}{ Dureza $(\mathrm{N})$} \\
\hline & $\left(\mathrm{L}^{*}\right)$ & $\left(a^{*}\right)$ & $\left(b^{*}\right)$ & $\left(\mathrm{L}^{*}\right)$ & $\left(a^{*}\right)$ & $\left(b^{*}\right)$ & \\
\hline Pão de linhaça padrão & $63 \pm 5^{\mathrm{a}}$ & $9 \pm 4^{\mathrm{a}}$ & $25 \pm 5^{\mathrm{a}}$ & $66 \pm 2^{\mathrm{a}}$ & $3 \pm 1^{a}$ & $13 \pm 1^{\mathrm{b}}$ & $2,8 \pm 0,2^{\mathrm{a}}$ \\
\hline Nova formulação com óleo de soja & $63 \pm 3^{\mathrm{a}}$ & $10 \pm 2^{\mathrm{a}}$ & $27 \pm 2^{\mathrm{a}}$ & $65 \pm 2^{\mathrm{a}}$ & $3 \pm 1^{\mathrm{a}}$ & $15 \pm 2^{\mathrm{a}}$ & $2,3 \pm 0,1^{b}$ \\
\hline
\end{tabular}

Letras iguais em uma mesma coluna indicam que não há diferença significativa entre as amostras $(\mathrm{p} \leq 0,05)$. $\mathrm{L}^{*}=$ luminosidade $\left(0=\right.$ preto; $100=$ branco); $\mathrm{a}^{*}=$ componente vermelhoverde; $\mathrm{e}^{*}=$ componente amarelo-azul. 
comparação com os da literatura, o que justifica a relevância dos dados aqui obtidos.

\section{Conclusões}

Os dados obtidos nesse trabalho permitiram verificar que é possível substituir a gordura hidrogenada por óleo de soja em pães de linhaça, obtendo formulações de maciez significativamente melhor.

A nova formulação com óleo de soja se destacou por apresentar quantidades significativamente maiores de ácidos graxos poliinsaturados e menores para os ácidos graxos trans. Esta nova formulação com óleo de soja, quando comparada com o pão de linhaça padrão, apresentou em sua porção os melhores valores diários de recomendação para os ácidos graxos ômega-6 e ômega-3.

A nova formulação com óleo de soja apresentou uma boa aceitação e foi representada positivamente pelos atributos maciez, textura e sabor. Portanto, a substituição da gordura hidrogenada por óleo de soja melhora o aporte nutricional e atende às características sensoriais do consumidor.

As análises obtidas trazem subsídios para atestar a importância do desenvolvimento de produtos nutricionalmente seguros, com bom paladar e com possibilidade de prevenir patologias.

\section{Referências bibliográficas}

ABNT - ASSOCIAÇÃO BRASILEIRA DE NORMAS TÉCNICAS. Especificações de cores de acordo com o sistema de notação Munsell - NBR 12694. Rio de Janeiro, nov. 1992.

ABI - American Institute of Baking. AIB Standard Procedure White Pan Bread Firmness Measurement. Disponível em: https://www.aibonline.org/research andtechnical/services/ prodqualityeval/AIBTextureAnalysis\%20Procedures.pdf. Acesso em: 17 Abril 2007.

ASCHERIO, A. et al. Trans fatty acids and coronary heart disease. New England Journal of Medicine, v. 340, n. 25, p. 1994-1998, 1999.

AUED-PIMENTEL, S. et al. Ácidos graxos saturados versus ácidos graxos trans em biscoitos. Revista Instituto Adolfo Lutz, v. 62, n. 2, p. 131-137, 2003.

BLIGH, E. G.; DYER, W. J. A rapid method of total lipid extraction and purification. Canadian Journal of Biochemistry and Physiology, v. 37, n. 8, p. 911-917, 1959.

BRASIL. Ministério da Saúde. Portaria n. 27, de 13 de janeiro de 1998. Regulamento técnico referente à informação nutricional complementar (declarações relacionadas ao conteúdo de nutrientes). Diário Oficial da República Federativa do Brasil, Brasília, DF, 16 jan. 1998, Seção 1, n. 11, p. 1-3.

BRASIL. Agência Nacional de Vigilância Sanitária. Resolução RDC n. 360, de 23 de dezembro de 2003. Regulamento Técnico sobre rotulagem nutricional de alimentos embalados. Diário Oficial da República Federativa do Brasil, Brasília, DF, 26 dez. 2003, Seção 1, p. 33-34.

CONNOR, W. E. Importance of n-3 fatty acids in health and diseases. American Journal of Clinical Nutrition, v. 71, n. 1, p. 171-75, 2000 .
CONNOR, W. E. n-3 Fatty acids from fish and fish oil: panacea or nostrum? American Journal of Clinical Nutrition, v. 74, n. 4, p. 415-416, 2001.

COVIngton, M. B. Omega-3 Fatty Acids. American Family Physician, v. 70, n. 1, p. 133-140, 2004.

CUNNANE, S. C. et al. High-linolenic acid flaxseed (Linum usitatissimum): some nutritional properties in humans. British Journal of Nutrition, v. 69, n. 2, p. 443-453, 1993.

CUNNANE, S. C. et al. Nutritional attributes of traditional flaxseed in healthy young adults. American Journal of Clinical Nutrition, v. 61, n. 1, p. 62-68, 1995.

CUNNIF, P. A. (Ed.). Official Methods of Analysis of AOAC international. 16th ed. Arlington: Association of Official Analytical Chemists, 1998. (CD-Rom).

DEPARTMENT OF HEALTH. Report on health and social subjects, Nutritional aspects of cardiovascular disease. London: HMSO, 1994. n. 46.

DRI's - Dietary Reference Intakes for Energy and Macronutrients. Institute of Medicine (IOM). Washington: National Academy Press, 2002. p. 107-264.

EMKEN, E. A. Nutrition and biochemistry of trans and positional fatty acid isomers in hydrogenated oils. Annual Review of Nutrition, v. 4, n. 4, p. 339-376, 1984.

FAO - Food and Agricultural Organization. Fats and oils in human nutrition. Table of contents, 1994. Disponível em: http://www.fao. org/docrep/v4700e/v4700e00.htm Acesso em: 20 July 2006.

FDA - Food and Drug Administration. 2003. Proposes new rules for trans fatty acids in nutrition labeling, nutrient content clains, and health clains. Disponível em: < http://www.gpoaccess.gov/ index.html>. Acesso em: 20 July 2006.

FELDMAN, E. B. et al. Position paper on trans fatty acids. American Journal of Clinical Nutrition, v. 63, n. 5, p. 663-670, 1996.

GIBSON, R. A.; MAKRIDES, M. n-3 polyunsaturated fatty acid requirements of term infants. American Journal of Clinical Nutrition, v. 71, n. 1, p. 251-255, 2000.

HALL III, C. A. et al. Stability of a-Linolenic Acid and Secoisolariciresinol Diglucoside in Flaxseed-Fortified Macaroni. Journal of Food Science, v. 70, n. 8, p. 483-489, 2005.

HUI, Y. H. Hidrogenation. In: HUI, Y. H. (Ed.). Bailey's industrial oil and fat products. New York: Editora Fereidoon Shahidi, 1996. p. 213-300.

IAL - INSTITUTO ADOLF LUTZ. Normas analíticas do Instituto Adolfo Lutz: Métodos Químicos e Físicos para Análise de Alimentos. 4 ed. São Paulo, 2005. p. 1018.

ISSFAL - International Society for the Study of Fatty Acids and Lipids. 2004. Recommendations for intake of Polyunsaturated fatty acids in Healthy adults. Disponível em: <http://www.issfal.org.uk/>. Acesso em: 20 July 2006.

JOSEPH, J. D.; ACKMAN, R. G. Capillary column gas chromatography method for analysis of encapsulated fish oil and fish oil ethyl esters: collaborative study. Journal Association of Official Analytical Chemists, v. 75, n. 3, p. 488-506, 1992.

JUDD, J. T. et al. Dietary trans fatty acids: effects of plasma lipids and lipoproteins on healthy men and women. American Journal of Clinical Nutrition, v. 59, n. 4, p. 861-868, 1994.

JUMP, D. B. The biochemistry of n-3 polyunsaturayed fatty acids. Journal of Biological Chemistry, v. 277, n. 11, p. 8755-8758, 2002. 
KENNY, S. et al. Incorporation of dairy ingredients into wheat bread: effects on dough rheology and bread quality. European Food Research and Technology, v. 210, n. 6, p. 391-396, 2000.

KRIS-ETHERTON, P.; HARRIS, W. S.; APPEL, L. J. Fish Consumption, Fish Oil, Omega-3 Fatty Acids, and Cardiovascular Disease. Circulation, v. 106, n. 21, p. 2747-2757, 2002.

LARQUE, E.; ZAMORA, S.; GIL, A. Dietary trans fatty acids in early life: a review. Early Human Development, v. 65, n. 2, p. 31-41, 2001.

LAWLESS, H. T.; HEYMANN, H. Acceptance and preference testing. In: LAWLESS, H. T.; HEYMANN, H. (Ed.). Sensory Evaluation of Food, Principles and Practices. New York: Chapman and Hall, 1998. p. 430-475.

LOUHERANTA, A. M. et al. A high-trans fatty acid diet and insulin sensitivity in young healthy women. Metabolism: clinical and experimental, v. 48, n. 7, p. 870-875, 1999.

MACHADO, F. M. S.; SANTIAGO, V. R. Os benefícios do consumo de alimentos funcionais. In: TORRES, E. A. F.; MACHADO, F. M. S. (Ed). Alimentos em questão: uma abordagem técnica para as dúvidas mais comuns. São Paulo: Ponto Crítico, 2001. p. 35-43.

MARTIN, C. A. et al. Trans Polynsaturated Fatty Acid Contents in Brazilian Refined Soybean Oil. Analytical Sciences, v. 22, n. 4, p. $631,2006$.

MARTIN, C. A. et al. Trans Polynsaturated Trans fatty acid content of Brazilian biscuits. Food Chemistry, v. 93, n. 3, p. 445-448, 2005.

MAYES, P. A. Metabolism of unsaturated fatty acids \& eicosanoids. In: MURRAY, R. K.; GRANNER, D. K.; MAYES, P. A.; RODWELL, V. W. (Ed). Harper Biochemistry. Stamford: Appleton \& Lange, 1996. p. 236-244.

OLIVEIRA, A. P. V. et al. Aceitação de sobremesas lácteas dietéticas e formuladas com açúcar: teste afetivo e Mapa de Preferência Interno. Ciência e Tecnologia de Alimentos, v. 24, n. 4, p. 627-633, 2004.

PINTO, E. P. et al. Características da batata frita em óleos com diferentes graus de insaturação. Boletim Centro de Pesquisa de Processamento de Alimentos, v. 21, n. 2, p. 293-302, 2003.
SANIBAL, E. A. A.; MANCINI FILHO, J. Perfil de ácidos graxos trans de óleo e gordura hidrogenada de soja no processo de fritura. Ciência e Tecnologia de Alimentos, v. 24, n. 1, p. 27-31, 2004.

SEBEDIO, J. L. et al. Formation of fatty acid geometrical isomers and of cyclic fatty acid monomers during the finish frying of frozen prefried potatoes. Food Research International, v. 29, n. 2, p. 109-116, 1996.

SRINIVASAN, C. S.; IRZ, X.; SHANKAR, B. An assessment of the potential consumption impacts of WHO dietary norms in OECD countries. Food Policy, v. 31, n. 1, p. 53-77, 2006.

STATISTICA. Statistica 6.0 Software. Tulsa: StatSoft, 2001.

STONE, H.; SIDEL, J. L. Sensory evaluation practices. 2 ed. London: Academic Press, 1993.

THURNHAM, D. I. Functional foods: cholesterol-lowering benefits of plants sterols. British Journal of Nutrition, v. 82, n. 4, p. 255-256, 1999.

VAINIO, H.; MUTANEM, M. Functional foods. Bluring the distinction between food and medicine. Scandinavian Journal of Work, Environment \& Health, v. 26, n. 2, p. 178-80, 2000.

VALENZUELA, A. B.; NIETO, K. S. Ácidos grasos omega-6 y omega-3 en la nutrición perinatal: su importancia en el desarrollo del sistema nervioso y visual. Revista Chilena de Pediatria, v. 74, n. 2, p. 149-157, 2003.

VALENZUELA, A. B.; MORGADO, N. Trans fatty acid isomers in human health and in the food industry. Biological Research, v. 32, n. 4, p. 273-287, 1999.

VOISEY, P. W. Instrumental measurements of food texture. In: DEMAN, J. M.; VOISEY, P. W.; RASPER, V. F.; STANLEY, D. W. Rheology and Texture in Food Quality. Westport: AVI, 1976. p. 79-141.

WHO - WORLD HEALTH ORGANIZATION. Nutrition. Science - Policy. 1995. WHO and FAO Joint Consultation: fats and oils in human nutrition. New York: Nutrition Reviews. Disponível em: <http://www.who.int/>. Acesso em: 20 July 2006. 